\title{
Dynamics of a Predator-Prey System with Beddington-DeAngelis Functional Response and Delays
}

\author{
Nai-Wei Liu and Ting-Ting Kong \\ School of Mathematics and Information Science, Yantai University, Yantai, Shandong 264005, China \\ Correspondence should be addressed to Nai-Wei Liu; liunaiwei@aliyun.com
}

Received 25 January 2014; Accepted 14 May 2014; Published 26 May 2014

Academic Editor: Xinguang Zhang

Copyright ( 2014 N.-W. Liu and T.-T. Kong. This is an open access article distributed under the Creative Commons Attribution License, which permits unrestricted use, distribution, and reproduction in any medium, provided the original work is properly cited.

We consider a predator-prey system with Beddington-DeAngelis functional response and delays, in which not only the stage structure on prey but also the delay due to digestion is considered. First, we give a sufficient and necessary condition for the existence of a unique positive equilibrium by analyzing the corresponding locations of a hyperbolic curve and a line. Then, by constructing an appropriate Lyapunov function, we prove that the positive equilibrium is locally asymptotically stable under a sufficient condition. Finally, by using comparison theorem and the $\omega$-limit set theory, we study the global asymptotic stability of the boundary equilibrium and the positive equilibrium, respectively. Also, we obtain a sufficient condition to assure the global asymptotic stability.

\section{Introduction}

The purpose of this paper is to consider the following predator-prey system with Beddington-DeAngelis functional response and delays:

$$
\begin{gathered}
x_{i}^{\prime}(t)=\tilde{a} x(t)-d_{i} x_{i}(t)-\tilde{a} e^{-d_{i} \tau_{1}} x\left(t-\tau_{1}\right), \\
x^{\prime}(t)=\widetilde{a} e^{-d_{i} \tau_{1}} x\left(t-\tau_{1}\right)-b x^{2}(t)-\frac{c x(t) y(t)}{m_{1}+m_{2} x(t)+m_{3} y(t)}, \\
y^{\prime}(t)=-d y(t)+\frac{f x\left(t-\tau_{2}\right) y\left(t-\tau_{2}\right)}{m_{1}+m_{2} x\left(t-\tau_{2}\right)+m_{3} y\left(t-\tau_{2}\right)},
\end{gathered}
$$

where $x_{i}(t)$ denotes the immature prey population density, $x(t)$ denotes the mature prey population density, and $y(t)$ denotes the mature predator population density, and all the parameters $\tau_{1}, \tau_{2}, \widetilde{a}, d_{i}, b, c, d, f, m_{1}, m_{2}$, and $m_{3}$ are positive. More precisely, the parameters $\tau_{1}$ and $\tau_{2}$ represent the time that the prey juveniles and the predator juveniles take to be mature, respectively. $\widetilde{a}$ is the intrinsic growth rate of the prey and $d_{i}$ is the death rate of immature preys. The term $\tilde{a} e^{-d_{i} \tau_{1}}$ denotes the surviving rate of the immature prey during the delay period to be mature. The constant $b$ denotes the intensity of intraspecific action on the prey and $d$ is the death rate of the predator. The function $c x(t) y(t) /\left(m_{1}+m_{2} x(t)+\right.$ $\left.m_{3} y(t)\right)$ represents the Beddington-DeAngelis functional response and the function $f x\left(t-\tau_{2}\right) y\left(t-\tau_{2}\right) /\left(m_{1}+m_{2} x(t-\right.$ $\left.\left.\tau_{2}\right)+m_{3} y\left(t-\tau_{2}\right)\right)$ represents the predator's growth rate that comes from the Beddington-DeAngelis functional response during time period $\left[t-\tau_{2}, t\right]$.

Actually, the predator-prey system with BeddingtonDeAngelis functional response has been extensively studied in the literature. In the original work, Beddington [1] and DeAngelis et al. [2] proposed the predator-prey system as

$$
\begin{gathered}
x^{\prime}(t)=a x(t-\tau)-b x^{2}(t)-\frac{c x(t) y(t)}{m_{1}+m_{2} x(t)+m_{3} y(t)}, \\
y^{\prime}(t)=-d y(t)+\frac{f x(t) y(t)}{m_{1}+m_{2} x(t)+m_{3} y(t)} .
\end{gathered}
$$

Shaikhet [3] considered the stability of a positive point of equilibrium of one nonlinear system with aftereffect and stochastic perturbations. Indeed, the author in [3] not only obtained sufficient conditions for asymptotic mean square stability of the considered systems, but also showed that the trivial solution is asymptotically mean square stable and the 
positive equilibrium is stable in probability by constructing appropriate Lyapunov functions. In particular, Shaikhet expounded a general method of construction of Lyapunov functions for stochastic functional differential equations and obtained the stability conditions for functional differential equations in [4]. See also [5-10] for the corresponding results about the predator's functional response. Particularly, Huo [8] studied the global attractiveness of delay diffusive prey-predator systems with the Michaelis-Menten functional response.

In the real world, many species usually have a life history that takes them through two stages, immature stage and mature stage. Recently, the system with the stage structure on prey has been extensively studied. In particular, She and Li [11] analyzed the following Beddington-DeAngelis model with the stage structure on prey:

$$
\begin{gathered}
x_{i}^{\prime}(t)=\tilde{a} x(t)-d_{i} x_{i}(t)-\tilde{a} e^{-d_{i} \tau} x(t-\tau), \\
x^{\prime}(t)=\tilde{a} e^{-d_{i} \tau} x(t-\tau)-b x^{2}(t)-\frac{c x(t) y(t)}{m_{1}+m_{2} x(t)+m_{3} y(t)}, \\
y^{\prime}(t)=-d y(t)+\frac{f x(t) y(t)}{m_{1}+m_{2} x(t)+m_{3} y(t)},
\end{gathered}
$$

where the stage structure was introduced by a constant time delay. In fact, it is the case of $\tau_{2}=0$ in system (1). See also [1215] for predator-prey models with Beddington-DeAngelis functional response and stage structure.

More recently, Xiang et al. [16] established a virus dynamics model with Beddington-DeAngelis functional response and delays. In [16], they not only incorporated the delay $\tau_{1}$, which described the time between viral entry into a target cell and the production of new virus particles, but also incorporated the delay $\tau_{2}$, which described the maturation time of the newly produced viruses. Additionally, it is well known that the growth of the predator is affected by the time delay due to digestion. This motivates us to resolve the issue.

Motivated by [16], in the present paper, we study the predator-prey system (1) with Beddington-DeAngelis functional response and delays, in which not only the stage structure on prey but also the delay due to digestion is considered.

More precisely, let $a=\tilde{a} e^{-d_{i} \tau_{1}}$ and assume that

$$
x_{i}(0)=\tilde{a} \int_{-\tau_{1}}^{0} e^{-d_{i} s} x(s) d s
$$

holds. Then, based on similar conclusions for Lemma 3.1 in [10], we obtain

$$
x_{i}(t)=\tilde{a} \int_{-\tau_{1}}^{0} e^{-d_{i} s} x(t+s) d s
$$

which means that $x_{i}(t)$ depends on $x(t)$ completely. Thus, we can obtain the following system, which is separated from (1):

$$
\begin{gathered}
x^{\prime}(t)=a x\left(t-\tau_{1}\right)-b x^{2}(t)-\frac{c x(t) y(t)}{m_{1}+m_{2} x(t)+m_{3} y(t)}, \\
y^{\prime}(t)=-d y(t)+\frac{f x\left(t-\tau_{2}\right) y\left(t-\tau_{2}\right)}{m_{1}+m_{2} x\left(t-\tau_{2}\right)+m_{3} y\left(t-\tau_{2}\right)} .
\end{gathered}
$$

It is obvious that, for studying the dynamics of the predator-prey system (1) with Beddington-DeAngelis functional response and delays, it is sufficient to study the dynamics of (6).

The rest of the organization of this paper is as follows. In Section 2, we first establish the local stability of the boundary equilibria of (6) by analyzing the characteristic equations, and then, we study the local asymptotic stability of the positive equilibria of (6) by constructing an appropriate Lyapunov function. In Section 3, we consider the global asymptotic stability of the boundary equilibrium and the positive equilibrium, respectively.

\section{Local Stability of Equilibria}

In this section, we study the local stability of the equilibria of (6). By direct calculations, we have that the system (6) has the origin equilibrium $E_{0}(0,0)$ and the boundary equilibrium $E_{1}((a / b), 0)$.

In order to investigate the existence of the positive equilibrium, we consider the equations

$$
\begin{gathered}
(a-b x)\left(m_{1}+m_{2} x+m_{3} y\right)-c y=0 \\
-d\left(m_{1}+m_{2} x+m_{3} y\right)+f x=0
\end{gathered}
$$

and obtain the following result.

Theorem 1. The system (6) has a unique positive equilibrium $E^{*}\left(x^{*}, y^{*}\right)$ if and only if the condition

$$
\left(f-d m_{2}\right) \frac{a}{b}>d m_{1}
$$

holds, which is equivalent to the condition

$$
\left(f-d m_{2}\right) \frac{\tilde{a} e^{-d_{i} \tau_{1}}}{b}>d m_{1} .
$$

Proof. We prove the existence of the positive equilibrium by analyzing (7). For the first equation of (7), it is easy to see that its corresponding curves go through the points $(a / b, 0)$, $\left(-m_{1} / m_{2}, 0\right)$, and $\left(0, a m_{1} /\left(c-a m_{3}\right)\right)$ when $c-a m_{3} \neq 0$. Furthermore, in case of $\left(\left(c-a m_{3}\right) / b m_{3}\right) \neq m_{1} / m_{2}$, the first equation is a hyperbolic equation and has two asymptotic lines $x+\left(\left(c-a m_{3}\right) / b m_{3}\right)=0$ and $y+\left(m_{2} / m_{3}\right) x+\left(\left(b m_{1} m_{3}-\right.\right.$ $\left.\left.c m_{2}\right) / b m_{3}^{2}\right)=0$. If $\left(\left(c-a m_{3}\right) / b m_{3}\right)=m_{1} / m_{2}$, the first equation can be simplified as $\left(m_{1}+m_{2} x\right)\left(a m_{2}-b m_{2} x-\right.$ $\left.b m_{3} y\right)=0$, which are two lines. 
For the second equation of (7), it implies a line which goes through the points $\left(0,-m_{1} / m_{3}\right)$ and $\left(d m_{1} /\left(f-d m_{2}\right), 0\right)$ when $d m_{1} /\left(f-d m_{2}\right) \neq 0$. Their corresponding figures are as shown in Figures 1, 2, and 3.

Consequently, by analyzing Figures 1, 2, and 3 and discussions, we obtain that (6) has a unique positive equilibrium.

Condition (9) indicates that the positive equilibrium $E^{*}$ exists for all $\tau_{1}$ in the interval $I=\left(0, \tau^{*}\right)$, where

$$
\tau^{*}=\frac{1}{d_{i}} \ln \frac{\widetilde{a}\left(f-d m_{2}\right)}{b d m_{1}} .
$$

Furthermore, $E^{*}$ will coincide with $E_{1}$ when $\tau_{1}=\tau^{*}$ and there exists no positive equilibrium for $\tau_{1}>\tau^{*}$.

Now, we study the stability of equilibria $E_{0}(0,0), E_{1}((a / b), 0)$ and $E^{*}\left(x^{*}, y^{*}\right)$, respectively.

At the beginning, we rewrite system (6) as $\bar{Z}^{\prime}(t)=$ $\bar{F}\left(\bar{Z}(t), \bar{Z}\left(t-\tau_{1}\right), \bar{Z}\left(t-\tau_{2}\right)\right)$, where $\bar{Z}(t)=(x(t), y(t)), \bar{Z}(t-$ $\left.\tau_{1}\right)=\left(x\left(t-\tau_{1}\right), y\left(t-\tau_{1}\right)\right), \bar{Z}\left(t-\tau_{2}\right)=\left(x\left(t-\tau_{2}\right), y\left(t-\tau_{2}\right)\right)$.
Then, for an arbitrary fixed point $\bar{Z}^{*}=(x, y)$, by letting $P=(\partial \bar{F} / \partial \bar{Z}(t))_{\bar{Z}^{*}}, Q=\left(\partial \bar{F} / \partial \bar{Z}\left(t-\tau_{1}\right)\right)_{\bar{Z}^{*}}$, and $R=$ $\left(\partial \bar{F} / \partial \bar{Z}\left(t-\tau_{2}\right)\right)_{\bar{Z}^{*}}$, we have

$$
\begin{gathered}
P=\left(\begin{array}{cc}
-2 b x-c q_{x}^{\prime} & -c q_{y}^{\prime} \\
0 & -d
\end{array}\right)_{\bar{X}^{*}}^{,} \quad Q=\left(\begin{array}{ll}
a & 0 \\
0 & 0
\end{array}\right)_{\bar{X}^{*}}, \\
R=\left(\begin{array}{cc}
0 & 0 \\
f q_{x}^{\prime} & f q_{y}^{\prime}
\end{array}\right)_{\bar{X}^{*}},
\end{gathered}
$$

where

$$
\begin{gathered}
q(x, y)=\frac{x y}{m_{1}+m_{2} x+m_{3} y}, \\
q_{x}^{\prime}=\frac{y\left(m_{1}+m_{3} y\right)}{\left(m_{1}+m_{2} x+m_{3} y\right)^{2}}, \\
q_{y}^{\prime}=\frac{x\left(m_{1}+m_{2} x\right)}{\left(m_{1}+m_{2} x+m_{3} y\right)^{2}} .
\end{gathered}
$$

Therefore, the characteristic equation of the system (6) at the point $\bar{Z}^{*}$ is as follows:

$$
\begin{aligned}
\left|P+Q e^{-\lambda \tau_{1}}+R e^{-\lambda \tau_{2}}-\lambda I\right| & =\left|\begin{array}{cc}
-2 b x-c q_{x}^{\prime}+a e^{-\lambda \tau_{1}}-\lambda & -c q_{y}^{\prime} \\
f q_{x}^{\prime} e^{-\lambda \tau_{2}} & -d+f q_{y}^{\prime} e^{-\lambda \tau_{2}}-\lambda
\end{array}\right| \\
& =\left|\begin{array}{cc}
-2 b x-\frac{c y\left(m_{1}+m_{3} y\right)}{\left(m_{1}+m_{2} x+m_{3} y\right)^{2}}+a e^{-\lambda \tau_{1}}-\lambda & -\frac{c x\left(m_{1}+m_{2} x\right)}{\left(m_{1}+m_{2} x+m_{3} y\right)^{2}} \\
\frac{f y\left(m_{1}+m_{3} y\right)}{\left(m_{1}+m_{2} x+m_{3} y\right)^{2}} e^{-\lambda \tau_{2}} & -d+\frac{f x\left(m_{1}+m_{2} x\right)}{\left(m_{1}+m_{2} x+m_{3} y\right)^{2}} e^{-\lambda \tau_{2}}-\lambda
\end{array}\right|=0 .
\end{aligned}
$$

Thus, according to the characteristic equation at the point $E_{0}$, we obtain the following result.

Theorem 2. The equilibrium $E_{0}(0,0)$ is unstable.

Proof. The characteristic equation of the system (6) at the point $E_{0}$ is

$$
\begin{aligned}
\left|P+Q e^{-\lambda \tau_{1}}+R e^{-\lambda \tau_{2}}-\lambda I\right|_{(0,0)} & =\left|\begin{array}{cc}
a e^{-\lambda \tau_{1}}-\lambda & 0 \\
0 & -d-\lambda
\end{array}\right| \\
& =(\lambda+d)\left(\lambda-a e^{-\lambda \tau_{1}}\right)=0 .
\end{aligned}
$$

Obviously, $\lambda=-d$ is a negative eigenvalue and the other eigenvalue depends on the solution of $\lambda-a e^{-\lambda \tau_{1}}=0$.

Noticing that the line $y=\lambda$ and the curve $y=a e^{-\lambda \tau_{1}}$ must intersect at a unique point $(\lambda, y)$, where $\lambda$ is a positive value, we can obtain that $E_{0}$ is a saddle. Thus, $E_{0}$ is unstable.

According to the characteristic equation of the point $E_{1}$, we have the following theorem.
Theorem 3. The equilibrium $E_{1}((a / b), 0)$ is unstable if $(f-$ $\left.d m_{2}\right)(a / b)>d m_{1}$ and is locally asymptotically stable if $(f-$ $\left.d m_{2}\right)(a / b)<d m_{1}$.

Proof. The characteristic equation of the system (6) at the point $E_{1}$ is

$$
\begin{aligned}
\mid P & +Q e^{-\lambda \tau_{1}}+R e^{-\lambda \tau_{2}}-\left.\lambda I\right|_{((a / b), 0)} \\
& =\left|\begin{array}{cc}
-2 a+a e^{-\lambda \tau_{1}}-\lambda & -\frac{a c}{b m_{1}+a m_{2}} \\
0 & -d+\frac{a f}{b m_{1}+a m_{2}} e^{-\lambda \tau_{2}}-\lambda
\end{array}\right| \\
& =\left(\lambda+2 a-a e^{-\lambda \tau_{1}}\right)\left(\lambda+d-\frac{a f}{b m_{1}+a m_{2}} e^{-\lambda \tau_{2}}\right) \\
& =0 .
\end{aligned}
$$

For the equation $\lambda+2 a-a e^{-\lambda \tau_{1}}=0$, it is clear that the line $y=\lambda+2 a$ and the curve $y=a e^{-\lambda \tau_{1}}$ must intersect at a unique point $(\lambda, y)$, where $\lambda$ is a negative value. And for the equation 


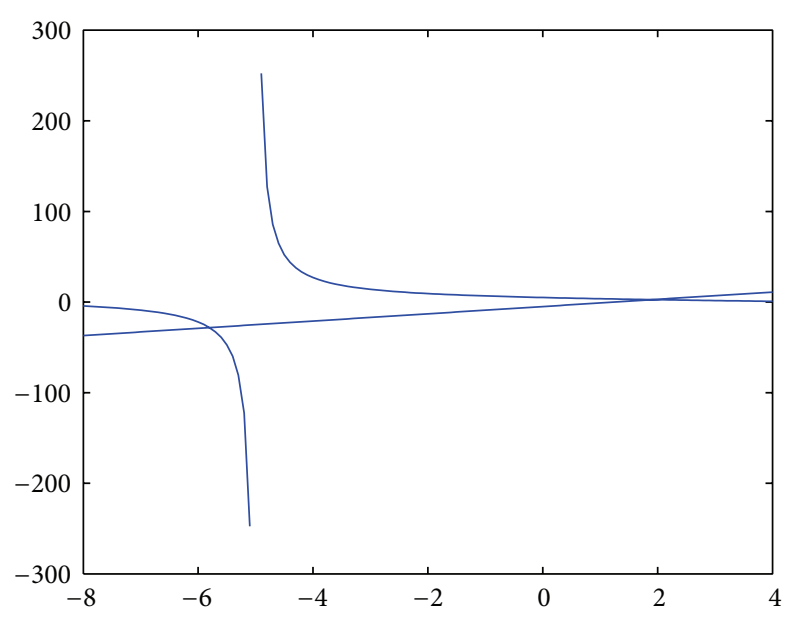

FIgURE 1: $-m_{1} / m_{2}<-\left(c-a m_{3}\right) / b m_{3}<0$.

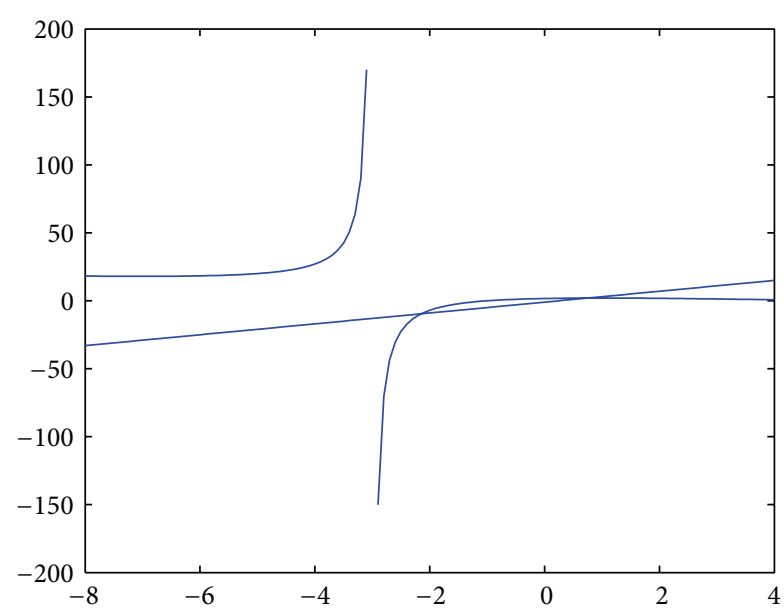

FIGURE 2: $-\left(c-a m_{3}\right) / b m_{3}<-m_{1} / m_{2}<0$.

$\lambda+d-\left(a f /\left(b m_{1}+a m_{2}\right)\right) e^{-\lambda \tau_{2}}=0$, noticing that the line $y=$ $\lambda+d$ and the curve $y=\left(a f /\left(b m_{1}+a m_{2}\right)\right) e^{-\lambda \tau_{2}}$ must intersect at a unique point $(\lambda, y)$, we have that $\lambda$ is positive when $(f-$ $\left.d m_{2}\right)(a / b)>d m_{1}$ and is negative when $\left(f-d m_{2}\right)(a / b)<$ $d m_{1}$. Consequently, $E_{1}$ is unstable if $\left(f-d m_{2}\right)(a / b)>d m_{1}$ and locally asymptotically stable if $\left(f-d m_{2}\right)(a / b)<d m_{1}$.

In the rest of this section, by constructing an appropriate Lyapunov function, we study the stability of the positive equilibrium $E^{*}\left(x^{*}, y^{*}\right)$ under condition (8). Let

$$
\begin{aligned}
& x(t)=x^{*}+u(t), \\
& y(t)=y^{*}+v(t)
\end{aligned}
$$

then, the linearization of the system (6) is

$$
\begin{gathered}
u^{\prime}(t)=A u\left(t-\tau_{1}\right)-B u(t)-C v(t), \\
v^{\prime}(t)=-D v(t)+E v\left(t-\tau_{2}\right)+F u\left(t-\tau_{2}\right),
\end{gathered}
$$

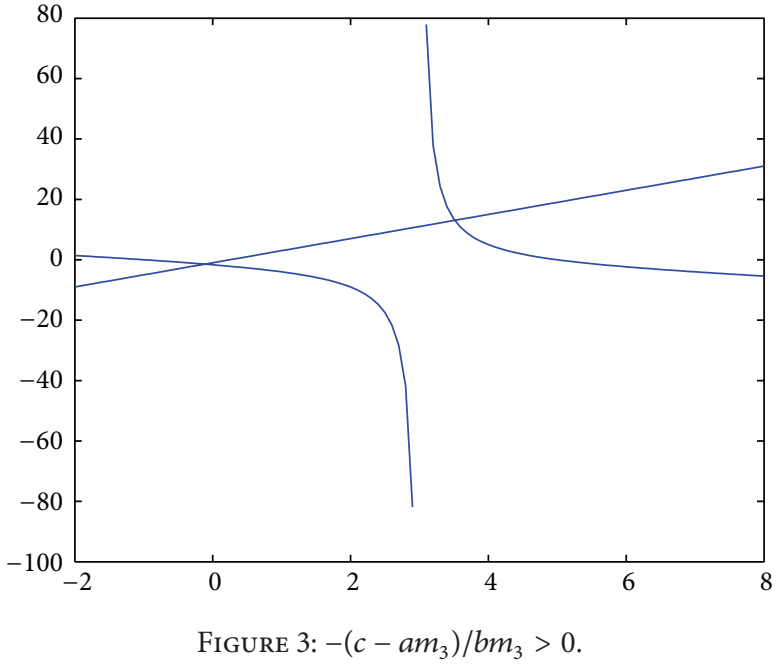

where

$$
\begin{gathered}
A=a, \quad B=2 b x^{*}+\frac{c y^{*}\left(m_{1}+m_{3} y^{*}\right)}{\left(m_{1}+m_{2} x^{*}+m_{3} y^{*}\right)^{2}}, \\
C=\frac{c x^{*}\left(m_{1}+m_{2} x^{*}\right)}{\left(m_{1}+m_{2} x^{*}+m_{3} y^{*}\right)^{2}}, \quad D=d, \\
E=\frac{f x^{*}\left(m_{1}+m_{2} x^{*}\right)}{\left(m_{1}+m_{2} x^{*}+m_{3} y^{*}\right)^{2}}, \\
F=\frac{f y^{*}\left(m_{1}+m_{3} y^{*}\right)}{\left(m_{1}+m_{2} x^{*}+m_{3} y^{*}\right)^{2}} .
\end{gathered}
$$

Obviously, all the constants defined above are positive, and the system (17) can be recast as

$$
\begin{aligned}
u^{\prime}(t)= & (A-B) u(t)-C v(t)-A \int_{t-\tau_{1}}^{t} u^{\prime}(s) d s \\
= & (A-B) u(t)-C v(t) \\
& -A \int_{t-\tau_{1}}^{t}\left[A u\left(s-\tau_{1}\right)-B u(s)-C v(s)\right] d s \\
v^{\prime}(t)= & (E-D) v(t)-E \int_{t-\tau_{2}}^{t} v^{\prime}(s) d s+F u(t) \\
& -F \int_{t-\tau_{2}}^{t} u^{\prime}(s) d s \\
= & (E-D) v(t)+F u(t) \\
& -F \int_{t-\tau_{2}}^{t}\left[A u\left(s-\tau_{1}\right)-B u(s)-C v(s)\right] d s \\
& -E \int_{t-\tau_{2}}^{t}\left[-D v(s)+E v\left(s-\tau_{2}\right)+F u\left(s-\tau_{2}\right)\right] d s .
\end{aligned}
$$


By constructing an appropriate Lyapunov function, we have the following theorem.

Theorem 4. Let $\tau_{1} \in\left(0, \tau^{*}\right)$ with $\tau^{*}$ defined in (10), and assume that

$$
\tau_{1}, \tau_{2} \in\left(0, \tau_{*}\right), \quad|F-C|<\min \{2(D-E), 2(B-A)\},
$$

also holds, where

$$
\begin{gathered}
\tau_{*}=\min \left\{\frac{2(D-E)-|F-C|}{A C+F(A+B+E)+2\left(C F+D E+E^{2}\right)},\right. \\
\left.\frac{2(B-A)-|F-C|}{A(2 A+2 B+C)+F(A+B+E)}\right\} .
\end{gathered}
$$

Then, the equilibrium $(0,0)$ of the system (17) is locally asymptotically stable, which implies that the positive equilibrium $E^{*}\left(x^{*}, y^{*}\right)$ of the system (6) is locally asymptotically stable.

Proof. To illustrate the equilibrium $(0,0)$ of the system (17) is locally asymptotically stable, it is sufficient to study the existence of a strict Lyapunov function. By letting $W_{11}(t)=$ $u^{2}(t)$ and differentiating $W_{11}(t)$ with respect to $t$, we have

$$
\begin{aligned}
W_{11}^{\prime}(t)= & 2(A-B) u^{2}(t)-2 C u(t) v(t) \\
& -2 A u(t) \int_{t-\tau_{1}}^{t}\left[A u\left(s-\tau_{1}\right)-B u(s)-C v(s)\right] d s \\
\leq & 2(A-B) u^{2}(t)-2 C u(t) v(t) \\
& +A(A+B+C) \tau_{1} u^{2}(t) \\
& +A \int_{t-\tau_{1}}^{t}\left[A u^{2}\left(s-\tau_{1}\right)+B u^{2}(s)+C v^{2}(s)\right] d s
\end{aligned}
$$

Letting $W_{12}(t)=A \int_{t-\tau_{1}}^{t} \int_{z}^{t}\left[A u^{2}\left(s-\tau_{1}\right)+B u^{2}(s)+C v^{2}(s)\right] d s d z$ and differentiating $W_{12}(t)$ with respect to $t$, we have

$$
\begin{aligned}
W_{11}^{\prime}(t)+W_{12}^{\prime}(t) \leq & 2(A-B) u^{2}(t)-2 C u(t) v(t) \\
& +A(A+B+C) \tau_{1} u^{2}(t) \\
& +A \tau_{1}\left[A u^{2}\left(t-\tau_{1}\right)+B u^{2}(t)+C v^{2}(t)\right] .
\end{aligned}
$$

Furthermore, by letting $W_{13}(t)=A^{2} \tau_{1} \int_{t-\tau_{1}}^{t} u^{2}(s) d s$ and $W_{1}(t)=W_{11}(t)+W_{12}(t)+W_{13}(t)$, we have

$$
\begin{aligned}
W_{1}^{\prime}(t) \leq & {\left[2(A-B)+A(2 A+2 B+C) \tau_{1}\right] u^{2}(t) } \\
& +A C \tau_{1} v^{2}(t)-2 C u(t) v(t) .
\end{aligned}
$$

Next, by letting $W_{21}(t)=v^{2}(t)$ and differentiating $W_{21}(t)$ with respect to $t$, we obtain

$$
\begin{aligned}
& W_{21}^{\prime}(t) \\
& =2(E-D) v^{2}(t)+2 F u(t) v(t)-2 v(t) \\
& \times \int_{t-\tau_{2}}^{t}\left\{F\left[A u\left(s-\tau_{1}\right)-B u(s)-C v(s)\right]\right. \\
& \left.+E\left[-D v(s)+E v\left(s-\tau_{2}\right)+F u\left(s-\tau_{2}\right)\right]\right\} d s \\
& \leq 2(E-D) v^{2}(t)+2 F u(t) v(t) \\
& +[F(A+B+C)+E(D+E+F)] \tau_{2} v^{2}(t) \\
& +\int_{t-\tau_{2}}^{t}\left\{F\left[A u^{2}\left(s-\tau_{1}\right)+B u^{2}(s)+C v^{2}(s)\right]\right. \\
& \left.+E\left[D v^{2}(s)+E v^{2}\left(s-\tau_{2}\right)+F u^{2}\left(s-\tau_{2}\right)\right]\right\} d s .
\end{aligned}
$$

Letting $W_{22}(t)=\int_{t-\tau_{2}}^{t} \int_{z}^{t}\left\{F\left[A u^{2}\left(s-\tau_{1}\right)+B u^{2}(s)+C v^{2}(s)\right]+\right.$ $\left.E\left[D v^{2}(s)+E v^{2}\left(s-\tau_{2}\right)+F u^{2}\left(s-\tau_{2}\right)\right]\right\} d s d z$ and differentiating $W_{22}(t)$ with respect to $t$, we have

$$
\begin{aligned}
W_{21}^{\prime}(t) & +W_{22}^{\prime}(t) \\
\leq 2 & (E-D) v^{2}(t)+2 F u(t) v(t) \\
+ & {[F(A+B+C)+E(D+E+F)] \tau_{2} v^{2}(t) } \\
+ & \left\{F\left[A u^{2}\left(t-\tau_{1}\right)+B u^{2}(t)+C v^{2}(t)\right]\right. \\
& \left.+E\left[D v^{2}(t)+E v^{2}\left(t-\tau_{2}\right)+F u^{2}\left(t-\tau_{2}\right)\right]\right\} \tau_{2} .
\end{aligned}
$$

By letting $W_{23}(t)=A F \tau_{2} \int_{t-\tau_{1}}^{t} u^{2}(s) d s, W_{24}(t)=$ $E^{2} \tau_{2} \int_{t-\tau_{1}}^{t} v^{2}(s) d s$, and $W_{25}(t)=E F \tau_{2} \int_{t-\tau_{2}}^{t} u^{2}(s) d s$, we have

$$
\begin{aligned}
& W_{23}^{\prime}(t)=A F \tau_{2}\left[u^{2}(t)-u^{2}\left(t-\tau_{1}\right)\right], \\
& W_{24}^{\prime}(t)=E^{2} \tau_{2}\left[v^{2}(t)-v^{2}\left(t-\tau_{2}\right)\right], \\
& W_{25}^{\prime}(t)=E F \tau_{2}\left[u^{2}(t)-u^{2}\left(t-\tau_{2}\right)\right] .
\end{aligned}
$$

By letting $W_{2}(t)=W_{21}(t)+W_{22}(t)+W_{23}(t)+W_{24}(t)+W_{25}(t)$, we have

$$
\begin{aligned}
W_{2}^{\prime}(t) & \\
\leq & \left\{2(E-D)+\tau_{2}\left[F(A+B+E)+2\left(C F+D E+E^{2}\right)\right]\right\} \\
& \times v^{2}(t)+F(A+B+E) \tau_{2} u^{2}(t)+2 F u(t) v(t) .
\end{aligned}
$$


Finally, we let $W(t)=W_{1}(t)+W_{2}(t)$. Obviously, $W(t) \geq 0$ and $W(t)=0$ if and only if $u(t)=v(t)=0$. Besides, when $u(t)=v(t)=0, W^{\prime}(t)=0$. Furthermore,

$$
\begin{aligned}
W^{\prime}(t) & \\
\leq & \left\{2(E-D)+A C \tau_{1}\right. \\
& \left.+\tau_{2}\left[F(A+B+E)+2\left(C F+D E+E^{2}\right)\right]\right\} \\
& \times v^{2}(t)+2(F-C) u(t) v(t) \\
& +\left[2(A-B)+A(2 A+2 B+C) \tau_{1}+F(A+B+E) \tau_{2}\right] \\
& \times u^{2}(t) \\
\leq & \left\{2(E-D)+A C \tau_{1}\right. \\
& \left.+\tau_{2}\left[F(A+B+E)+2\left(C F+D E+E^{2}\right)\right]\right\} \\
& \times v^{2}(t)+2|F-C||u(t)||v(t)| \\
& +\left[2(A-B)+A(2 A+2 B+C) \tau_{1}+F(A+B+E) \tau_{2}\right] \\
& \times u^{2}(t) \\
\leq & \left\{2(E-D)+|F-C|+A C \tau_{1}\right. \\
& \left.+\tau_{2}\left[F(A+B+E)+2\left(C F+D E+E^{2}\right)\right]\right\} v^{2}(t) \\
+ & {\left[2(A-B)+|F-C|+A(2 A+2 B+C) \tau_{1}\right.} \\
& \left.+F(A+B+E) \tau_{2}\right] u^{2}(t) .
\end{aligned}
$$

Based on the conditions in Theorem 4, we have $W^{\prime}(t)<0$ if $u^{2}(t)+v^{2}(t)>0$. Thus, $W(t)$ is a strict Lyapunov function. According to the Lyapunov stability theorem, the equilibrium $(0,0)$ of the system $(17)$ is locally asymptotically stable, which implies that the positive equilibrium $E^{*}\left(x^{*}, y^{*}\right)$ of the system (6) is locally asymptotically stable.

\section{Global Stability of Equilibria}

In this section, we study the global asymptotic stability of the boundary equilibrium $E_{1}((a / b), 0)$ and the positive equilibrium $E^{*}\left(x^{*}, y^{*}\right)$, respectively. We first give two lemmas which will be used later.

Lemma 5 (see [10, Lemma 3.2]). For a given system

$$
x^{\prime}(t)=b_{1} x(t-\tau)-a_{1} x(t)-a_{2} x^{2}(t),
$$

where $b_{1}>0, a_{1} \geq 0, a_{2}>0, \tau \geq 0$, and $x(t)>0$ for all $-\tau \leq t \leq 0$, we have

(i) $\lim _{t \rightarrow+\infty} x(t)=\left(b_{1}-a_{1}\right) / a_{2}$ if and only if $b_{1}>a_{1}$;

(ii) $\lim _{t \rightarrow+\infty} x(t)=0$ if and only if $b_{1} \leq a_{1}$.

Lemma 6 (see [11, Lemma 3.2]). Let $S=\{(x, y): x>0, y>$ $0\}$ and $\bar{S}=\{(x, y): x \geq 0, y \geq 0\}$. Then, the sets $S$ and $\bar{S}$ are both invariant sets.
Based on Lemmas 5 and 6, we prove that the boundary equilibrium $E_{1}$ is globally attractive.

Theorem 7. Let $(x(t), y(t))$ be the solution of (6) with positive initial condition in $S$.

(i) If $\left(f-d m_{2}\right)(a / b)<d m_{1}$, then $E_{1}((a / b), 0)$ is globally attractive.

(ii) If $\left(f-d m_{2}\right)(a / b)>d m_{1}$, then $E_{1}((a / b), 0)$ is unstable.

Proof. If $\left(f-d m_{2}\right)(a / b)<d m_{1}$, we first prove that $\lim _{t \rightarrow+\infty} y(t)=0$.

Due to the first equation (6), there is

$$
x^{\prime}(t) \leq a x\left(t-\tau_{1}\right)-b x^{2}(t)
$$

In view of Lemma 6 and by considering the following comparison equation

$$
p^{\prime}(t)=a p\left(t-\tau_{1}\right)-b p^{2}(t), \quad p(0)=x(0)>0,
$$

we have $x(t) \leq p(t)$ for all $t \geq 0$. In addition, by Lemma 5 , we obtain that $\lim _{t \rightarrow+\infty} p(t)=(a / b)$. Thus, there exists a sufficiently small positive constant $\varepsilon$ with $\left(f-d m_{2}\right)((a / b)+$ $\varepsilon)<d m_{1}$ such that, for this $\varepsilon$, there exists a $T_{\varepsilon}>0$ such that $x(t)<(a / b)+\varepsilon$ for all $t>T_{\varepsilon}$. Substituting it into the second equation of (6), we have

$$
y^{\prime}(t) \leq-d y(t)+\frac{f((a / b)+\varepsilon)}{m_{1}+m_{2}((a / b)+\varepsilon)} y\left(t-\tau_{2}\right),
$$

for all $t>T_{\varepsilon}$. Considering the following comparison equation:

$$
\begin{gathered}
q^{\prime}(t)=-d q(t)+\frac{f((a / b)+\varepsilon)}{m_{1}+m_{2}((a / b)+\varepsilon)} q\left(t-\tau_{2}\right), \\
q\left(T_{\varepsilon}+\tau_{2}\right)=y\left(T_{\varepsilon}+\tau_{2}\right)>0,
\end{gathered}
$$

and letting $q(t)=e^{\lambda t}$, we have

$$
e^{\lambda t}\left[\lambda+d-\frac{f((a / b)+\varepsilon)}{m_{1}+m_{2}((a / b)+\varepsilon)} e^{-\lambda \tau_{2}}\right]=0 .
$$

Then the line $y=\lambda+d$ and the curve $y=\left(f((a / b)+\varepsilon) /\left(m_{1}+\right.\right.$ $\left.\left.m_{2}((a / b)+\varepsilon)\right)\right) e^{-\lambda \tau_{2}}$ must intersect at a unique point $(\lambda, y)$. Since $\left(f-d m_{2}\right)((a / b)+\varepsilon)<d m_{1}$, we can obtain that $d>$ $f((a / b)+\varepsilon) /\left(m_{1}+m_{2}((a / b)+\varepsilon)\right)$. Thus, we deduce that $\lambda<0$. Therefore, $\lim _{t \rightarrow+\infty} q(t)=\lim _{t \rightarrow+\infty} e^{\lambda t}=0$.

Obviously, based on the comparison theorem, we get that $y(t) \leq q(t)$ for all $t \geq T+\tau_{2}$. Thus, $\lim _{t \rightarrow+\infty} y(t)=0$.

Next, we prove that $x(t) \rightarrow(a / b)$ as $t \rightarrow+\infty$. 
Since $\lim _{t \rightarrow+\infty} y(t)=0$, for any given $\varepsilon_{1} \in(0,(a / b))$, there exists a $T_{1}>0$ such that $0<y(t)<\left(b m_{1} / 2 c\right) \varepsilon_{1}$ for all $t \geq T_{1}$. Thus, we have

$$
\begin{aligned}
a x\left(t-\tau_{1}\right)-b x^{2}(t) \geq & x^{\prime}(t) \\
= & a x\left(t-\tau_{1}\right)-b x^{2}(t) \\
& -\frac{c x(t) y(t)}{m_{1}+m_{2} x(t)+m_{3} y(t)} \\
\geq & a x\left(t-\tau_{1}\right)-b x^{2}(t)-\frac{c x(t) y(t)}{m_{1}} \\
\geq & a x\left(t-\tau_{1}\right)-b x^{2}(t)-\frac{b \varepsilon_{1}}{2} x(t),
\end{aligned}
$$

for all $t \geq T_{1}$. That is,

$$
\begin{aligned}
a x\left(t-\tau_{1}\right)-b x^{2}(t) & \geq x^{\prime}(t) \\
& \geq a x\left(t-\tau_{1}\right)-b x^{2}(t)-\frac{b \varepsilon_{1}}{2} x(t) .
\end{aligned}
$$

Then, we consider the following comparison equation:

$$
\begin{gathered}
\widetilde{p}^{\prime}(t)=a \tilde{p}\left(t-\tau_{1}\right)-b \widetilde{p}^{2}(t)-\frac{b \varepsilon_{1}}{2} \widetilde{p}(t), \\
\widetilde{p}\left(T_{1}\right)=x\left(T_{1}\right)>0 .
\end{gathered}
$$

Since $a>b \varepsilon_{1}$, based on Lemma 5, we have that $\lim _{t \rightarrow+\infty} \widetilde{p}(t)=(a / b)-\left(\varepsilon_{1} / 2\right)$. Additionally, we have that $\widetilde{p}(t) \leq x(t) \leq p(t)$ for all $t \geq T_{1}$.

Since $\lim _{t \rightarrow+\infty} p(t)=(a / b)$, for the above $\varepsilon_{1}$, there exists a $T_{2}>0$ such that $p(t) \leq(a / b)+\varepsilon_{1}$ for all $t>T_{2}$. Similarly, since $\lim _{t \rightarrow+\infty} \tilde{p}(t)=(a / b)-\left(\varepsilon_{1} / 2\right)$ for the above $\varepsilon_{1}$, we also get that there exists a $T_{3}>0$ such that $\tilde{p}(t)-(a / b)+\left(\varepsilon_{1} / 2\right)>$ $-\left(\varepsilon_{1} / 2\right)$ for all $t>T_{3}$. Therefore, if we let $T_{0}=\max \left\{T_{1}, T_{2}, T_{3}\right\}$, there is $-\varepsilon_{1}<x(t)-(a / b)<\varepsilon_{1}$ for all $t>T_{0}$. Thus, we have $\lim _{t \rightarrow+\infty} x(t)=(a / b)$.

At last, we prove the second part of this theorem. We can assure that $\left(f-d m_{2}\right)(a / b)>d m_{1}$ and try to derive a contradiction. If $\left(f-d m_{2}\right)(a / b)>d m_{1}$, then (6) has a unique positive equilibrium $\left(x^{*}, y^{*}\right)$ by Theorem 1 , which is also a solution of (6); that is, $\left(x^{*}, y^{*}\right)=\left(x^{*}(t), y^{*}(t)\right)$ satisfies (6) and satisfies $\lim _{t \rightarrow+\infty}\left(x^{*}(t), y^{*}(t)\right)=((a / b), 0)$ by the above arguments, which contradicts the fact that $\left(x^{*}, y^{*}\right)$ is a positive equilibrium. Thus, conclusion (ii) holds. This completes the proofs.

Remark 8. In the above theorem, we derive the global attractiveness of the boundary equilibrium $E_{1}((a / b), 0)$. Unfortunately, we just obtain a sufficient condition that $(f-$ $\left.d m_{2}\right)(a / b)<d m_{1}$ to ensure that $E_{1}$ is globally asymptotically stable. If $\left(f-d m_{2}\right)(a / b)=d m_{1}$, since the second equation of (6) is a delay diffusion equation, we cannot derive that $\lim _{t \rightarrow+\infty} y(t)=0$ by using comparison theorem. We leave it for a further study.
Furthermore, for the first equation of (1), we can solve explicitly as

$$
\lim _{t \rightarrow+\infty} x_{i}(t)=\frac{a^{2}\left(e^{d_{i} \tau_{1}}-1\right)}{b d_{i}}
$$

Thus, by Theorem 7, we can obtain the following corollary directly.

Corollary 9. Let $\left(x_{i}(t), x(t), y(t)\right)$ be the solution of (1) with the positive initial condition. If $f \leq d m_{2}$, then

$$
\lim _{t \rightarrow+\infty}\left(x_{i}(t), x(t), y(t)\right)=\left(\frac{a^{2}}{b d_{i}}\left(e^{d_{i} \tau_{1}}-1\right), \frac{a}{b}, 0\right) .
$$

In the rest of this section, we will study the global attractiveness of the positive equilibrium $E^{*}$ and obtain the following result.

Theorem 10. Assume that (8) and the following conditions

$$
a m_{3}>c, \quad \frac{f\left(a m_{3}-c\right)}{b m_{1} m_{3}+m_{2}\left(a m_{3}-c\right)}>d,
$$

hold, and let $(x(t), y(t))$ be the solution of (6) with the positive initial condition in $S$ such that $\lim _{t \rightarrow+\infty}(x(t), y(t))=E^{*}$. Then, the positive equilibrium $E^{*}$ of (6) is globally attractive.

Proof. Provided that conditions (8) and (41) hold, based on comparison theorem and by similar arguments in [11], we can obtain that, for any solution $(x(t), y(t))$ of $(6)$ with the positive initial condition in $S$, there exists a point $\bar{E}^{*}\left(\bar{x}^{*}, \bar{y}^{*}\right)$ such that $\lim _{t \rightarrow+\infty}(x(t), y(t))=\left(\bar{x}^{*}, \bar{y}^{*}\right)$. Indeed, just by modifying the coefficients of (6), the discussion in [11] also holds. We omit the proof here and refer to [11] for details.

Then, in view of the property of the $\omega$-limit set in [17] and by the uniqueness result of the positive equilibrium in Theorem 1, we have $\bar{E}^{*}=E^{*}$. Thus, we derive that $E^{*}$ is globally attractive.

\section{Conclusion}

In the present paper, we considered the stability of the equilibria of a predator-prey system with Beddington-DeAngelis functional response and delays. More precisely, we derived a sufficient and necessary condition for the existence of a unique positive equilibrium and proved that the positive equilibrium is locally asymptotically stable under a sufficient condition. Also, we established the local stability of the boundary equilibria by analyzing the characteristic equations. Finally, by using comparison theorem and the $\omega$-limit set theory, we studied the global asymptotic stability for both the boundary equilibrium and the positive equilibrium.

In particular, in Theorem 7 , we derived the global attractiveness of the boundary equilibrium $E_{1}((a / b), 0)$. But unfortunately, we just obtained a sufficient condition that $\left(f-d m_{2}\right)(a / b)<d m_{1}$ to ensure that $E_{1}$ was globally asymptotically stable. If $\left(f-d m_{2}\right)(a / b)=d m_{1}$, since the second equation of (6) was a delay diffusion equation, we 
could not derive that $\lim _{t \rightarrow+\infty} y(t)=0$ by using comparison theorem.

Moreover, as pointed out in [11], there are two interesting problems about system (1), which are related to the phenomena of bifurcations and the existence of periodic orbits of (1). We leave them for a further study.

\section{Conflict of Interests}

The authors declare that there is no conflict of interests regarding the publication of this paper.

\section{Acknowledgments}

The first author was partially supported by NSFC (11201402, 11371179 ) and NSF of Shandong Province of China (ZR2010AQ006). The second author was partially supported by Graduate Innovation Foundation of Yantai University (GIFYTU).

\section{References}

[1] J. R. Beddington, "Mutual interference between parasites or predators and its effect on searching efficiency," Journal of Animal Ecology, vol. 44, pp. 331-340, 1975.

[2] D. L. DeAngelis, R. A. Goldstein, and R. V. O'Neil, "A model of trophic interaction," Ecology, vol. 56, pp. 881-892, 1975.

[3] L. Shaikhet, "Stability of a positive point of equilibrium of one nonlinear system with aftereffect and stochastic perturbations," Dynamic Systems and Applications, vol. 17, no. 1, pp. 235-253, 2008.

[4] L. Shaikhet, Lyapunov Functionals and Stability of Stochastic Functional Differential Equations, Springer, New York, NY, USA, 2013.

[5] S. B. Hsu, S. P. Hubbell, and P. Waltman, "Competing predators," SIAM Journal on Applied Mathematics, vol. 35, no. 4, pp. 617625, 1978.

[6] H.-F. Huo, W.-T. Li, and J. J. Nieto, "Periodic solutions of delayed predator-prey model with the Beddington-DeAngelis functional response," Chaos, Solitons \& Fractals, vol. 33, no. 2, pp. 505-512, 2007.

[7] H.-F. Huo, "Periodic solutions for a semi-ratio-dependent predator-prey system with functional responses," Applied Mathematics Letters, vol. 18, no. 3, pp. 313-320, 2005.

[8] H.-F. Huo, "Permanence and global attractivity of delay diffusive prey-predator systems with the Michaelis-Menten functional response," Computers \& Mathematics with Applications, vol. 49, no. 2-3, pp. 407-416, 2005.

[9] T.-W. Hwang, "Global analysis of the predator-prey system with Beddington-DeAngelis functional response," Journal of Mathematical Analysis and Applications, vol. 281, no. 1, pp. 395401, 2003.

[10] S. Liu, L. Chen, and Z. Liu, "Extinction and permanence in nonautonomous competitive system with stage structure," Journal of Mathematical Analysis and Applications, vol. 274, no. 2, pp. 667-684, 2002.

[11] Z. She and H. Li, "Dynamics of a density-dependent stagestructured predator-prey system with Beddington-DeAngelis functional response," Journal of Mathematical Analysis and Applications, vol. 406, no. 1, pp. 188-202, 2013.
[12] C.-Y. Huang, Y.-J. Li, and H.-F. Huo, "The dynamics of a stage-structured predator-prey system with impulsive effect and Holling mass defence," Applied Mathematical Modelling, vol. 36, no. 1, pp. 87-96, 2012.

[13] H. Li and Y. Takeuchi, "Dynamics of the density dependent predator-prey system with Beddington-DeAngelis functional response," Journal of Mathematical Analysis and Applications, vol. 374, no. 2, pp. 644-654, 2011.

[14] H. Li, "Persistence in non-autonomous Lotka-Volterra system with predator-prey ratio-dependence and density dependence," Applied Mathematics, vol. 2, no. 9, pp. 1148-1153, 2011.

[15] H. Li and R. Zhang, "Stability and optimal harvesting of a delayed ratio-dependent predator-prey system with stage structure," Journal of Biomathematics, vol. 23, no. 1, pp. 40-52, 2008.

[16] H. Xiang, L.-X. Feng, and H.-F. Huo, "Stability of the virus dynamics model with Beddington-DeAngelis functional response and delays," Applied Mathematical Modelling, vol. 37, no. 7, pp. 5414-5423, 2013.

[17] W. Hahn, Stability of Motion, Springer, New York, NY, USA, 1967. 


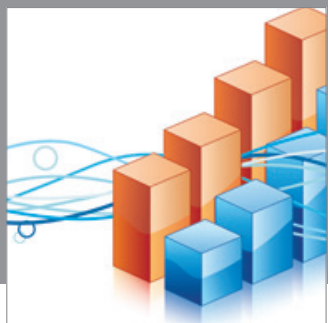

Advances in

Operations Research

mansans

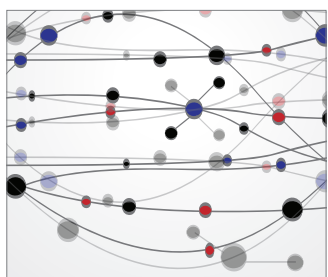

The Scientific World Journal
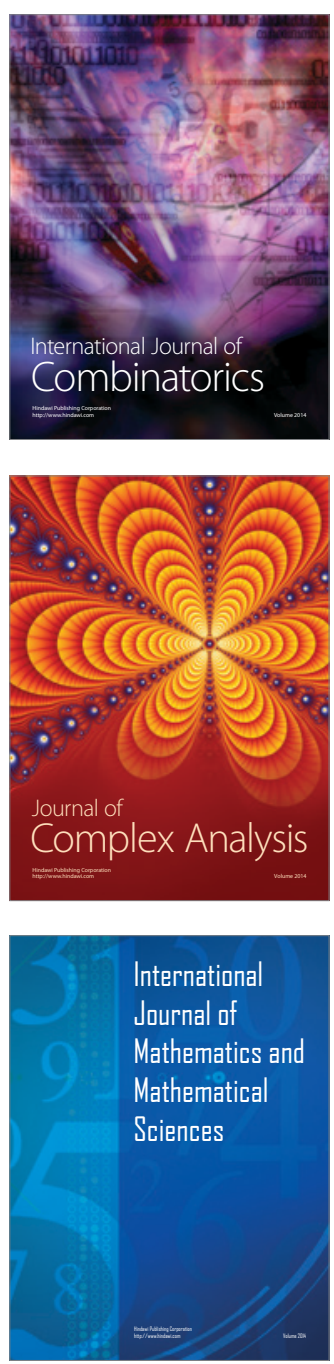
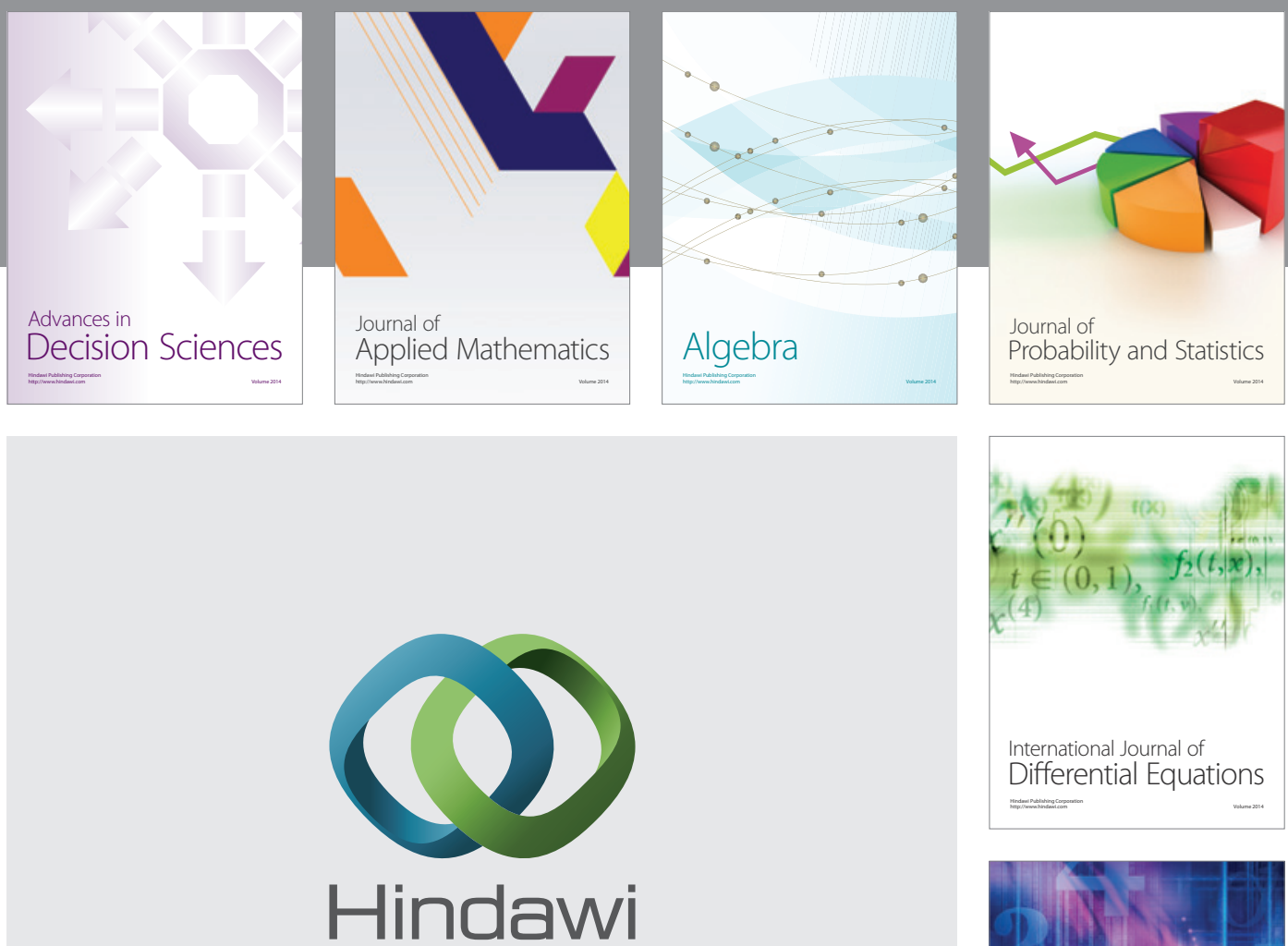

Submit your manuscripts at http://www.hindawi.com
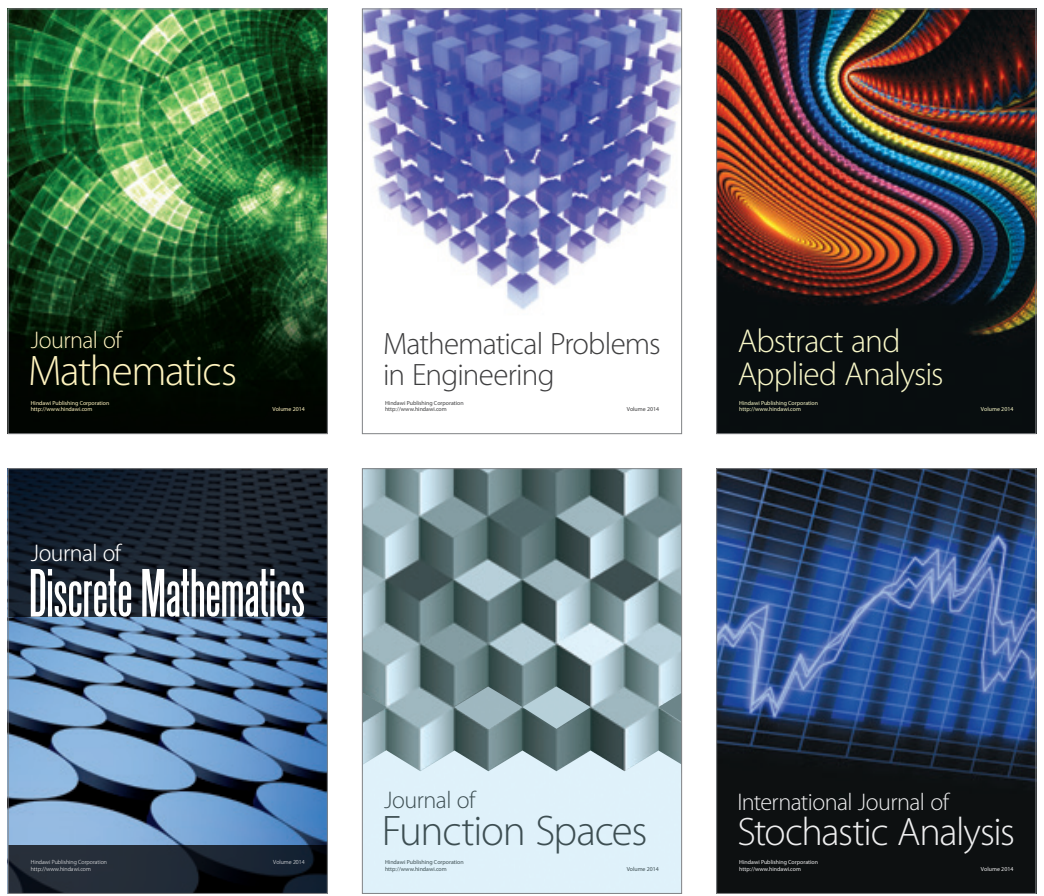

Journal of

Function Spaces

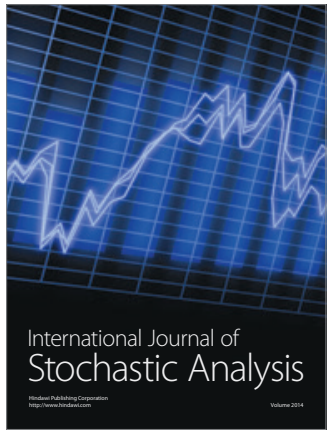

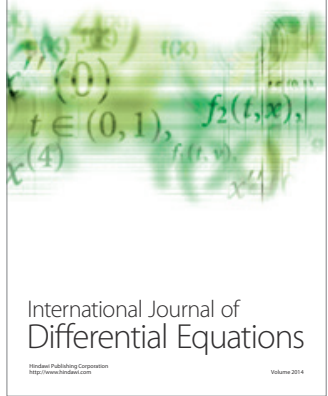
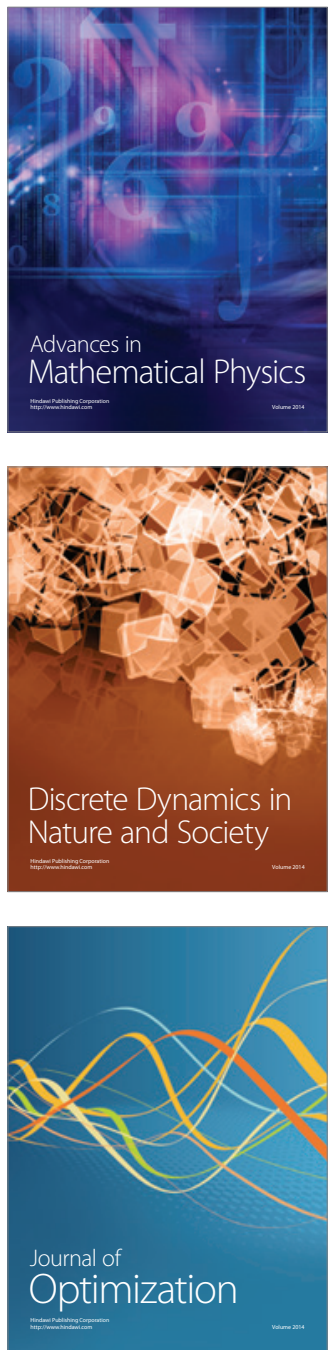